\title{
LA LEALTAD DEL JOVEN RESIDENTE A TRAVÉS DE LAS VARIABLES QUE LE VINCULAN CON SU DESTINO TURÍSTICO
}

\author{
Eduardo Parra López \\ José Alberto Martínez González. \\ Universidad de La Laguna
}

\section{RESUMEN}

En un contexto de turismo doméstico responsable y sostenible este trabajo tiene el objetivo de analizar el papel del apego al lugar, la familiaridad y la identidad de lugar en la formación de la lealtad del joven residente. La lealtad se define como la intención de visitar nuevamente el propio destino de residencia o de recomendarlo a otras personas. En el estudio no se incluyen aquellas variables de la empresa o del producto que son tradicionalmente tenidas en cuenta en el estudio de la formación de la lealtad en el sector turístico. Se constata que los niveles de todas las variables son altos y se genera un modelo causal que explica la formación de la lealtad exclusivamente a partir de las variables mencionadas. Aunque el análisis discriminante demuestra que no existen diferencias significativas en las respuestas de los jóvenes residentes en función del sexo, de la isla de residencia o de la cantidad de viajes realizados en el propio destino, en el trabajo se pone de manifiesto que sí existen algunas diferencias por razones de sexo en determinadas relaciones causales del modelo estructural propuesto.

Palabras clave: Turismo doméstico, lealtad del turista, apego al lugar, familiaridad, identidad de lugar, satisfacción del turista.

Recibido: 24 de marzo de 2015

Devuelto para su revisión: 10 de julio de 2015

Aceptado: 12 de enero de 2016

Departamento de Dirección de Empresas e Historia Económica. Universidad da La Laguna. 38071 TENERIFE (España).E-mail: eparra@ull.es, jmartine@ull.edu.es

Cátedra de Turismo-CajaCanarias-ASHOTEL 


\title{
Loyalty of young residents through variables that link them to their own tourist destination
}

\begin{abstract}
Within the context of sustainable and responsible domestic tourism, the aim of this paper is to analyze the role of place attachment, familiarity and place identity in loyalty formation among young residents in an island tourist destination. Loyalty is defined as the intention to visit a destination again or recommend it to others. However, variables related to tourism firms and products traditionally used in tourism loyalty research are not included in this study. Results show that levels of all variables analyzed are high and a causal model is developed that explains loyalty formation exclusively using them. Although discriminatory analysis shows that there are no significant differences in responses from young residents in terms of their sex, island of residence or number of visits made to the destination, but there are some differences shown regarding sex in certain, specific causal relations of the proposed structural model.
\end{abstract}

Keywords: Domestic tourist, tourist loyalty, place identity, place attachment, familiarity, tourist satisfaction.

\section{INTRODUCCIÓN}

El turismo tiene una creciente importancia debido a los efectos directos e indirectos (positivos y negativos) que, en un contexto de desarrollo turístico sostenible y responsable, ejerce a nivel económico, social y medioambiental, tanto en el ámbito local como global. A esto hay que añadir el hecho de que el turismo haya sufrido los efectos de la crisis en menor medida que otros sectores y haya supuesto un "colchón" amortiguador para infinidad de empresas, debido principalmente a la interrelaciones que el turismo mantiene con dichos sectores (Sun, Chi y Xu, 2013). A nivel económico y en el caso de España, el destino ocupa a nivel internacional el segundo lugar en términos de ingresos y la tercera posición por llegadas de viajeros internacionales (Sánchez, García y Marchante, 2014).

Ahora bien, a pesar del buen comportamiento del sector turístico las empresas del sector realizan grandes esfuerzos para conseguir la lealtad del consumidor, es decir, para que los turistas regresen nuevamente y recomienden el destino turístico a otras personas. Ello es debido a la creciente competencia de los mercados y a las mayores exigencias de los consumidores (Lee, 2013). Este proceso es especialmente importante en el caso de los destinos que se encuentran en la etapa de estancamiento o de madurez de su ciclo de vida, como es el caso de muchos destinos turísticos masivos de sol y playa (Oreja, Parra y Yanes, 2008).

En este contexto de lealtad se está produciendo en muchos destinos un interesante fenómeno que beneficia prácticamente a todos los stakeholders. En las regiones y destinos en los que se ha adoptado el turismo como una estrategia de desarrollo económico, como es el caso de España y más concretamente el de las Islas Canarias, los residentes se han 
convertido en turistas fieles a la propia región, o a regiones próximas dentro del país. Esto es debido en parte a la promoción que realizan las empresas turísticas, pero sobre todo como una alternativa a la crisis, de modo que los destinos se han convertido en "lugares refugio" (Wang y Xu, 2015). Este hecho está constatado por las estadísticas del Instituto de Estudios Turísticos de España, según las cuales el crecimiento del turismo residente es superior al $12 \%$ anual, siendo además la satisfacción y la lealtad de los residentes muy elevada (superior a 8,5 puntos sobre 10).

El interés por los residentes es alto porque ellos asumen el rol de turistas en el propio destino, contribuyendo con ello a aumentar los ingresos y a disminuir el carácter estacional de la demanda turística, facilitando la sostenibilidad económica de las empresas turísticas de la región. Pero los residentes en el destino también pueden aportar información de gran valor y contribuir a identificar y a generar los cambios deseados (Almeida, Balbuena y Cortés, 2015). Por otra parte, el residente adopta el rol de habitante del lugar, conviviendo con los turistas y enriqueciendo la experiencia y la integración del visitante. También asume el rol de trabajador en las empresas turísticas locales, favoreciendo la estancia del turista en el establecimiento alojativo (Gutiérrez, 2010).

Se han llevado a cabo múltiples investigaciones sobre los residentes de los destinos turísticos, y en ellas se han tenido en cuenta aspectos sociales, económicos y medioambientales. Infinidad de variables y prácticamente todos las metodologías de investigación se han aplicado en este campo. Sin embargo, los estudios acerca del turismo de los residentes en el propio destino son muy escasos (Draper, Woosnam y Norman, 2011). Esto es incomprensible si tenemos en cuenta que los resultados de los trabajos llevados a cabo sobre los residentes han puesto de manifiesto que sus percepciones positivas conllevan efectos positivos para su satisfacción y su lealtad, del mismo modo que lo contrario también ha sido constatado (Laszlo, Sherman y Ellison, 2005).

Por otra parte, en numerosos trabajos se pone de manifiesto que el desarrollo turístico sostenible y responsable debe realizarse pensando en los jóvenes residentes, que serán los encargados de llevar a cabo el relevo generacional (Jaafar, Noor y Rasoolimanesh, 2015). Los jóvenes constituyen un segmento que ha sido escasamente abordado en la literatura sobre turismo, lo cual es sorprendente teniendo en cuenta que el segmento representa un porcentaje importante de la población, que los jóvenes poseen un gran potencial de compra e influencia, y que son consumidores de productos turísticos, deportivos y de ocio, de una manera más autónoma a partir de los 18 años de edad (Wu y Pearce, 2013).

Se ha prestado una significativa atención al papel de los residentes en el desarrollo turístico en destinos consolidados y en destinos emergentes (Sharpley, 2014). Sin embargo es muy difícil encontrar referencias al estudio de los jóvenes residentes en destinos formados por islas. Esto no deja de ser llamativo si consideramos que los destinos turísticos insulares se caracterizan por una mayor vulnerabilidad social y ambiental a los impactos del desarrollo turístico, tanto o más que otros destinos (Scheyvens y Momsen, 2008). Por otra parte, la cercanía entre las distintas islas que forman el destino, las peculiaridades de cada una de ellas y la existencia de familiares y personas conocidas en dichas islas pueden favorecer el turismo de los residentes en el propio destino.

Respecto a la lealtad del turista, ésta se ha estudiado teniendo en cuenta variables relacionadas con la empresa (reputación, confianza, orientación al mercado), con el 
producto-destino (imagen, valor percibido, calidad) y con el propio turista (satisfacción, sexo, nacionalidad). Sin embargo son escasos los estudios en los que se analicen solamente y de manera conjunta variables que relacionan y vinculan al joven residente con el propio destino. Este es el caso del apego al lugar, la familiaridad y la identidad de lugar, un conjunto de variables que están muy relacionadas con la duración de la estancia y que pueden tener un papel relevante en las decisiones y en la lealtad del joven residente que decide hacer turismo en su propio destino turístico (Lee, Graefe y Burns, 2007).

Para responder a todas estas inquietudes y llamamientos este trabajo tiene por objeto el estudio de la formación de la lealtad del joven residente en el desempeño su rol como turista en el propio destino. En primer lugar se presentará el marco teórico sobre la formación lealtad en el ámbito turístico y seguidamente se expondrá la investigación llevada a cabo. Además del análisis descriptivo, el estudio supone la elaboración y validación de un modelo causal de formación de la lealtad del joven residente en el que sólo intervienen, además de la satisfacción, el apego, la familiaridad y la identidad de lugar, sin incluir variables de la empresa o del producto turístico.

\section{LA LEALTAD DEL TURISTA}

En la literatura se constata que la lealtad del consumidor es una variable estratégica en turismo, por los beneficios que aporta a las empresas y a otros stakeholders. La mayor parte de los estudios sobre la lealtad en el turismo son relativamente recientes y en general se han centrado en identificar los factores que la incrementan y en los beneficios que proporciona (Yuksel, Yuksel y Bilim, 2009).

Respecto a las definiciones de la lealtad, algunos autores han sugerido que no existe una única definición del constructo, sino tres (McKercher, Denizci y Ng, 2012). La lealtad vertical conllevaría que los turistas fueran fieles a un determinado elemento constitutivo del sistema turístico (una agencia de viajes o un destino turístico, por ejemplo). En el caso de la lealtad horizontal el turista puede ser fiel a más de un intermediario en un mismo nivel del canal de distribución (i. e. lealtad a varios hoteles). Por último, por medio de la lealtad experiencial el turista es fiel a un determinado estilo de vacaciones, y no a otros (el golf, el esquí). En este trabajo la lealtad del joven residente se relaciona con el sentido vertical del término, pues la formación de la lealtad se analiza específicamente en el destino turístico de las Islas Canarias.

Las definiciones de la lealtad inicialmente aportadas por la literatura estaban asociadas a la actitud positiva o de rechazo del consumidor respecto a un producto turístico, pero actualmente y en lo que respecta a este artículo el constructo se refiere a la voluntad declarada por el turista de repetir la compra en el futuro o de recomendar el producto (Zhang, Fu, Cai y Lu, 2014). Adicionalmente, frente a las consideraciones unidimensionales de la lealtad hoy en día se admite que el constructo es multidimensional, es decir, posee contenido afectivo, cognitivo y conativo (Forgas, Palau, Sánchez y Callarisa, 2012). Los aspectos cognitivos y afectivos de la lealtad son tenidos en cuenta en este trabajo porque en el mismo se analiza el papel del apego, la familiaridad y la identidad de lugar, variables con contenido cognitivo-afectivo. El carácter conativo de la lealtad 
se haya presente en este artículo en la propia definición y medición del constructo, pues se concibe como intención de repetir la compra o de recomendar el destino turístico.

Existe una serie de factores que hacen difícil definir, medir y gestionar la lealtad. En primer lugar se debe reconocer que el turismo es una industria basada en servicios intangibles que en muchos casos conlleva la intervención de diferentes destinos, culturas e intermediarios, todo lo cual conlleva un riesgo para el consumidor (Um, Chon y Ro, 2006). También se ha de considerar que aspectos tales como el carácter nacional o internacional del viaje, la distancia del trayecto, la fase del ciclo de vida del destino turístico, el historial de repetición, la relación del turista con las empresas o la nacionalidad del turista pueden condicionar la lealtad (McKercher y Denizci, 2010). Además, la repetición del viaje puede estar más relacionada con la costumbre que con factores verdaderamente asociados a la lealtad. A esto hay que añadir que, debido al carácter no lineal de la relación entre la satisfacción y la lealtad, muchos turistas satisfechos declaran que no volverán a visitar el destino o que no lo recomendarían (Pearce y Kang, 2009). Y en otros casos ha sido la repetición de métodos y conceptos en el estudio de la lealtad lo que ha impedido traspasar las fronteras conceptuales, metodológicas y prácticas en el estudio de la lealtad (McKercher, Denizci y Ng, 2012).

En los trabajos de investigación llevados a cabo sobre la formación de la lealtad en turismo (cuadro 1) se destaca la influencia de variables relacionadas con la empresa, con el producto-destino turístico y con el turista.

\section{Cuadro 1}

\section{VARIABLES QUE INFLUYEN EN LA FORMACIÓN DE LA LEALTAD}

\begin{tabular}{|l|l|l|}
\hline Grupo de variables & Variables & Autores \\
\hline \multirow{4}{*}{ De la empresa turística } & Orientación al mercado & Kohli y Jaworski (1990) \\
\cline { 2 - 3 } & Confianza & Kassim y Abdullah (2010) \\
\cline { 2 - 3 } & Reputación & Carmeli y Tishler (2005) \\
\hline \multirow{3}{*}{ Del producto o el destino turístico } & Calidad percibida & Um, Chon y Ro (2006) \\
\cline { 2 - 3 } & Valor percibido & Gupta y Kim (2010) \\
\cline { 2 - 3 } & Imagen & Bigné, Sánchez y Sánchez (2001) \\
\hline \multirow{2}{*}{ Relacionadas con el turista } & Satisfacción & Chi y Qu (2008) \\
\cline { 2 - 3 } & Personales (i. e. edad) & Woodside y Lysonski (1989) \\
\hline
\end{tabular}

Fuente: elaboración propia a partir de la revisión de la literatura.

La mayor parte de los modelos causales explicativos de la formación de la lealtad en turismo son similares al de la figura 1. En ellos se considera que la orientación al mercado es una de las principales variables que origina la cadena de efectos directos e indirectos que culmina en la satisfacción y la lealtad. La orientación al mercado por parte de la empresa conlleva adoptar una filosofía de satisfacción de necesidades del cliente y realizar actuaciones para conseguirlo (Bigné, Sánchez y Sánchez, 2001). Está 
comprobado que la orientación al mercado favorece la confianza, la responsabilidad y la reputación de la propia empresa (Meydeu y Lado, 2003). También mejora la imagen, la calidad y el valor percibido del producto o del destino, entendiendo por valor percibido la diferencia entre los beneficios y los sacrificios que para el turista tiene la compra o el consumo del producto (Narver y Slater, 1990). A su vez las variables mencionadas, que están relacionadas con la empresa y con el producto turístico, influyen en la formación de la satisfacción y, a través de ésta, de la lealtad (Andrades, Sánchez y Pulido, 2013). En la mayor parte de los trabajos se demuestra que la satisfacción, concebida como el grado en que se cumplen las expectativas del turista, es la variable que más directa y significativamente influye sobre la lealtad, mientras que las demás variables tienen una influencia indirecta a través de la satisfacción (Lee, Graefe y Burns, 2007; Chi y Qu, 2008; Wong y Wan, 2013).

\section{Figura 1 \\ MODELO GENERAL DE FORMACIÓN DE LA LEALTAD}

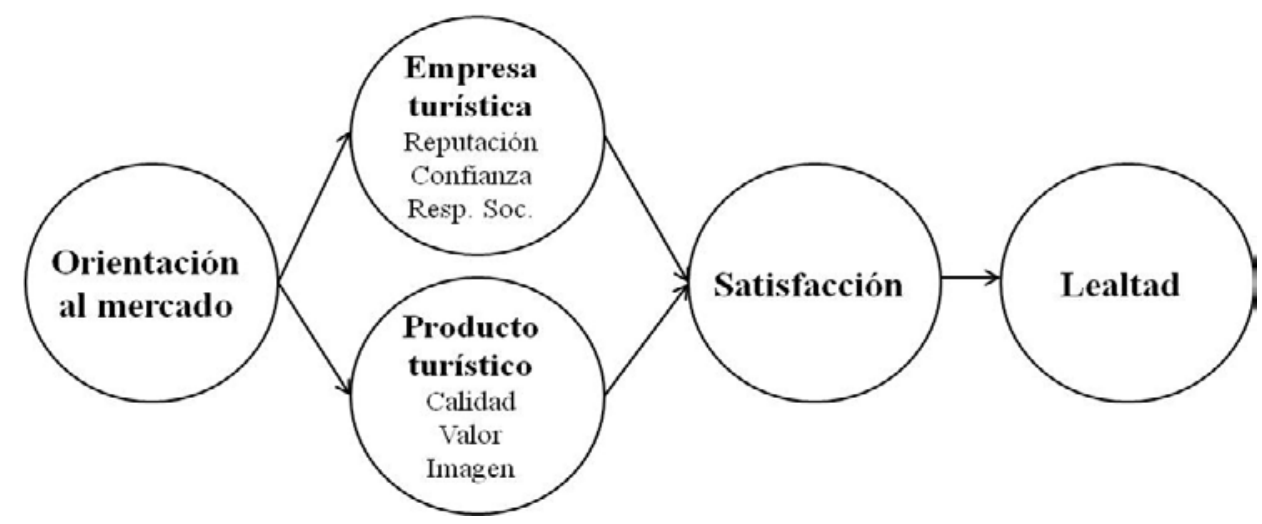

Fuente: elaboración propia a partir de la revisión de la literatura.

\section{EL PAPEL DEL APEGO AL LUGAR, LA FAMILIARIDAD Y LA IDENTIDAD EN LA FORMACIÓN DE LA LEALTAD}

Además de la influencia de las variables relacionadas con la empresa y con el producto-destino en la formación de la lealtad, se ha de tener en cuenta que los turistas son leales a los lugares que les ofrecen algún tipo de ventaja o beneficio, como puede ser la estabilidad, la seguridad, el confort o el sentimiento de pertenencia. No todos esos beneficios son siempre ofrecidos por la empresa o por el producto, o al menos no de una manera suficiente y adecuada (Hidalgo y Hernández, 2001). En el caso concreto de los residentes la lealtad tiene que ver sobre todo con los vínculos cognitivos, afectivos y sociales relacionados, entre otros aspectos, con la duración de la estancia. Este es precisamente uno de los elementos comunes del apego al lugar, la familiaridad y la identidad de lugar (Rollero y De Piccoli, 2010). Sin embargo, a pesar de su importancia, 
los estudios sobre dichas variables son relativamente recientes, y no es clara la diferencia entre los tres constructos ni tampoco está claro el papel de la empresa sobre dichas variables (Kyle, Graefe, Manning y Bacon, 2004a, 2004b; Lewicka, 2008).

En la formación del apego al lugar predomina el vínculo emocional del sujeto con el lugar (Yuksel, Yuksel y Bilim, 2009). Los turistas se apegan a un lugar cuando tienen posibilidades de expresarse a sí mismos, cuando el lugar es relevante para su estilo de vida, cuando las experiencias son positivas y, sobre todo, cuando la estancia es duradera (Hernández, Hidalgo, Salazar-Laplace y Hess, 2007). Respecto a los efectos del apego, éste es un predictor de la calidad percibida, de la satisfacción y de la lealtad (Hwang, Lee y Chen, 2005; Insch y Florek, 2008). Está demostrado que los sujetos con alto apego al lugar lo describen de manera positiva, mientras que los sujetos con menos apego lo describen de manera negativa (Rollero y De Piccoli, 2010). No obstante, existe confusión entre la identidad y el apego al lugar: mientras que para algunos autores los constructos son sinónimos, para otros son conceptos distintos, siendo la correlación entre ambas variables tan elevada como la discrepancia (Hernández, Hidalgo, SalazarLaplace y Hess, 2007).

La familiaridad es un estado subjetivo relacionado con la experiencia previa, el conocimiento y el aprendizaje sobre un determinado destino turístico, y sobre aspectos tan diversos como las atracciones del destino, la alimentación, el lenguaje y la cultura (Baloglu, 2001). La familiaridad es un antecedente de la imagen del destino, de la intención de viajar, de la satisfacción y de la lealtad (Kim, Ferrín y Rao, 2008). Los sujetos que poseen más familiaridad estarán menos dispuesto a elegir destinos turísticos alternativos (Lee, Graefe y Burns, 2007). Estos individuos resisten adecuadamente las interferencias de los estados emocionales y las opiniones de otras personas en el desarrollo de sus propios juicios. Ellos evalúan menos atributos del producto, tienden a realizar menos esfuerzo para reducir el riesgo percibido y tienen respuestas de compra más automatizadas. Además, mientras los sujetos menos familiarizados prefieren utilizar recursos y variables externas en sus decisiones de compra, como son la reputación y la imagen, los sujetos más familiarizados preferirán recursos internos, como es el caso de la satisfacción (Luo, Feng y Cai, 2008).

La identidad de lugar obedece a un proceso complejo y especialmente cognitivo por el cual, debido a la interacción del sujeto con el lugar, la persona se describe a sí misma en términos de pertenencia a dicho lugar ("soy español", "soy neoyorquino") (Rollero y De Piccoli, 2010). La identidad de lugar constituye una dimensión de la identidad social y personal que, como en el caso del apego y de la familiaridad, influye en la generación de conexiones, emociones, ideas y actitudes que pueden a su vez repercutir en el comportamiento, la satisfacción y la lealtad del turista. De este modo los sujetos cuya identidad de lugar se asocia a un destino turístico concreto estarán menos dispuesto a elegir destinos turísticos alternativos al planificar sus viajes (Lee, Graefe y Burns, 2007).

En este contexto, el modelo propuesto en este trabajo (figura 2) hace referencia a la influencia del apego, la familiaridad y la identidad en la formación de la lealtad, es decir, en la intención del joven residente de repetir el viaje en el propio destino o de recomendar su visita a otras personas. Dicha influencia sobre la lealtad puede ser directa o indirectamente a través de la satisfacción, que actuaría como variable mediadora. 


\section{Figura 2 \\ MODELO PROPUESTO DE FORMACIÓN DE LA LEALTAD}

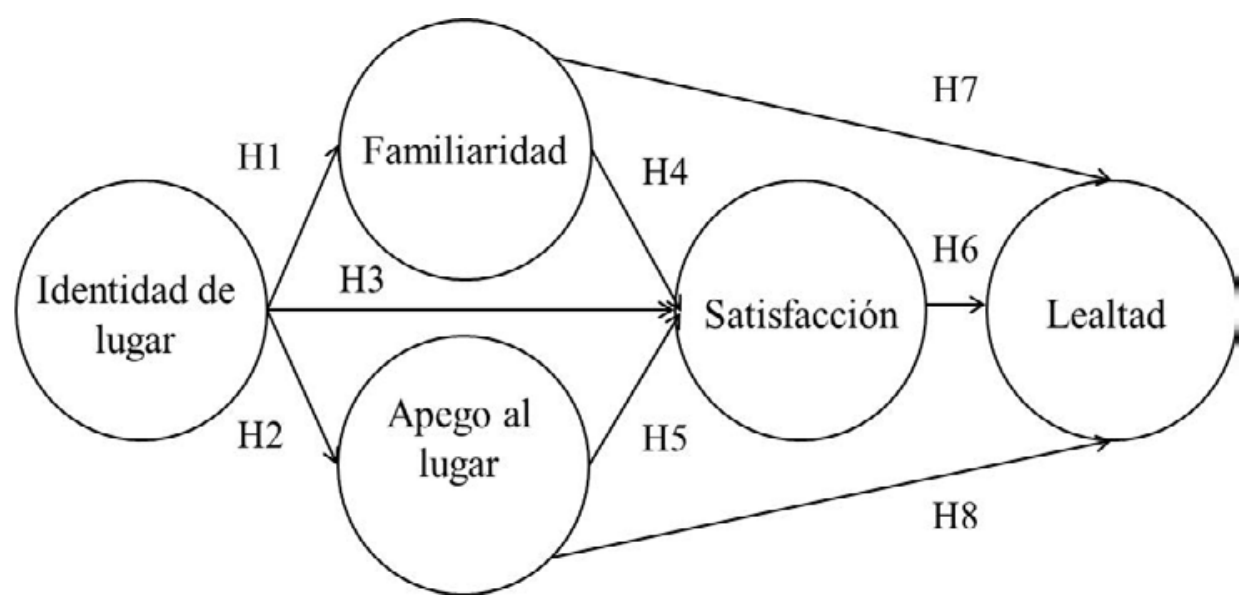

Fuente: elaboración propia a partir de la revisión de la literatura.

El modelo no incluye ninguna de las variables tradicionalmente estudiadas relativas a la empresa turística (orientación al mercado, reputación, confianza, responsabilidad) ni al producto (calidad, valor, imagen), pues en este trabajo se pretende demostrar que la lealtad del joven residente en el ejercicio de su rol como turista en su propio destino se puede conseguir exclusivamente a través de las variables que vinculan a dicho residente con el propio destino: el apego al lugar, la familiaridad y la identidad. Puede parecer evidente que la situación económica actual, la cercanía, el conocimiento del propio destino, así como la existencia de lazos de amistad o las propias circunstancias de los jóvenes residentes puedan influir en el crecimiento del turismo doméstico y en la formación de la lealtad de los jóvenes residentes. También es comprensible que los residentes posean altos niveles de apego, familiaridad e identidad en relación a su propio destino turístico, por la duración de la estancia y las experiencias vividas en el destino. Sin embargo, en este trabajo se analiza la relación causal del apego al lugar, la familiaridad y la identidad en la formación de la lealtad, además de los niveles declarados por los jóvenes respecto a dichas variables, no se analiza el papel de la crisis económica o de otros procesos en la formación de la lealtad.

En el modelo propuesto se acepta que la identidad de lugar es diferente del apego y de la familiaridad, tal y como proponen algunos autores (Jorgensen y Stedman, 2001), siendo la identidad la variable que origina la cadena de efectos que desemboca en la formación de la lealtad del joven residente. Teniendo esto en cuenta se han desarrollado las dos primeras hipótesis:

H1. La identidad de lugar influye directamente y de manera positiva en la formación de la familiaridad 
H2. La identidad de lugar repercute directamente en el apego al lugar

También se acepta la relación significativa, directa y positiva de la identidad de lugar con la satisfacción (Scott y Vitartas, 2008), del mismo modo que sucede con la familiaridad (Kim, Ferrín y Rao, 2008) y el apego al lugar (Insch y Florek, 2008). A raíz de ello se explicitaron las tres hipótesis siguientes:

H3. La identidad de lugar influye de manera directa en la satisfacción

H4. El apego repercute directamente en la formación de la satisfacción

H5. La familiaridad influye de manera directa en la satisfacción

Tal y como se constata en la literatura, en el modelo propuesto se parte de la base de que la satisfacción es la variable que más peso ejerce en la formación de la lealtad (Kozak, 2003), aunque también se asume en el modelo que la familiaridad (Gross y Brown, 2008) y el apego (Hwang, Lee y Chen, 2005) influyen directamente en la lealtad. De este modo se elaboraron las siguientes hipótesis:

H6. La satisfacción es la variable que más peso directo ejerce en la formación de la lealtad del joven residente

H7. La familiaridad influye de manera directa en la lealtad

H8. El apego al lugar repercute de manera directa en la formación de la lealtad

En el trabajo se tienen en cuenta dos cuestiones adicionales. En primer lugar, tal y como pone de manifiesto en los datos estadísticos sobre turismo, se parte de la base de que los jóvenes turistas residentes poseen un alto nivel de satisfacción y lealtad (Exceltur-Gobierno de Canarias, 2013). Estos datos nos llevaron a plantear la siguiente hipótesis:

H9. Los niveles de identidad, apego, familiaridad, satisfacción y lealtad de los jóvenes residentes son elevados

Por último, en numerosos estudios sociológicos se evidencia que las generaciones se ven influidas por similares factores socio-culturales y poseen patrones homogéneos cognitivos, afectivo y comportamentales (Charters et al., 2011). Este es el caso de los jóvenes de 18 y 19 años que forman parte de la población residente, un segmento claramente definido e integrante de la Generación "Y" que toma sus decisiones por haber llegado a la mayoría de edad (Nusair, Parsa y Cobanoglu, 2011). Con esta premisa la siguiente hipótesis establece que:

H10. No existen diferencias significativas en las respuestas de los jóvenes residentes por razones de sexo, de isla de residencia y de cantidad de viajes realizados en el propio destino, y tampoco por razones de sexo en las relaciones causales del modelo estructural propuesto. 


\section{METODOLOGíA}

Con el objeto de poder contrastar las hipótesis presentadas en este trabajo se ha llevado a cabo una aproximación cuantitativa en esta investigación que es descriptiva y predictivocausal.

El contexto del estudio ha sido el destino turístico de las Islas Canarias, un destino de sol y playa, maduro y masivo que ha atravesado todas las fases de su ciclo de vida. La región tiene algo más de dos millones de habitantes y aproximadamente el 50\% de su población se concentra en las dos islas que ostentan la capitalidad de las dos provincias que forman el archipiélago, y en las cuales se ha llevado a cabo el estudio: Tenerife y Gran Canaria. No es sencillo encontrar estudios de los residentes en destinos turísticos formados por islas. Este hecho, además de las posibilidades de extrapolar los resultados a otros destinos, sugieren que es necesario prestar una mayor atención a los destinos insulares (Oreja, Parra y Yanes, 2008).

La muestra final estuvo compuesta por 678 sujetos (cuadro 2). Inicialmente la muestra incluía un mayor número de sujetos, pero fueron excluidos aquellos que respondieron al cuestionario dando la misma puntuación a todos los ítems, o los que dejaron ítems sin responder. El tamaño de la muestra supera las diez veces el número de variables o de ítems, en concordancia con la "regla de las diez veces" propuesta por Chin (1998a) y Hair, Ringle y Sarstedt (2011) cuando se utilizan ecuaciones estructurales (PLS). El tamaño de la muestra también está en concordancia con trabajos recientes en los que se estudia la percepción del joven residente (Gutiérrez, 2010; Jaafar, Noor y Rasoolimanesh, 2015). Respecto a la edad, el 98\% de la muestra tenía una edad de 18 y 19 años.

\section{Cuadro 2}

\section{DESCRIPCIÓN DE LA MUESTRA}

\begin{tabular}{|l|c|c|c|c|c|c|c|c|}
\hline \multirow{2}{*}{} & \multicolumn{4}{|c|}{ Tenerife } & \multicolumn{4}{c|}{ Gran Canaria } \\
\cline { 2 - 9 } & $1^{\mathrm{o}}$ & $2^{\mathrm{o}}$ & Total & $\%$ & $1^{\circ}$ & $2^{\circ}$ & Total & $\%$ \\
\hline Hombres & 75 & 49 & 124 & $42 \%$ & 103 & 44 & 147 & $38 \%$ \\
\hline Mujeres & 106 & 62 & 168 & $58 \%$ & 143 & 96 & 239 & $62 \%$ \\
\hline Total & 181 & 111 & 292 & $100 \%$ & 246 & 140 & 386 & $100 \%$ \\
\hline
\end{tabular}

Fuente: elaboración propia.

La muestra se seleccionó de manera intencional para que estuviera formada por jóvenes residentes en las dos provincias de la región (Tenerife y Gran Canaria), consiguiendo así una mayor representatividad geográfica. Para poder realizar comparaciones entre sujetos de ambas provincias y asegurar la homogeneidad de las dos sub-muestras se seleccionó a estudiantes de primero y segundo curso que estudiaban la misma titulación en cada una de las dos universidades de la región. Hemos tenido en cuenta en este trabajo que los estudiantes universitarios constituyen un segmento que ha sido considerado en otros trabajos sobre turismo de destinos (Eurico, da Silva y do Valle, 2015). 
Adicionalmente, el carácter socio-cultural y generacional del estudio sugiere que la muestra utilizada es representativa, pues los jóvenes de 18 y 19 años de edad forman parte de la denominada Generación "Y", que comparte similares procesos cognitivos, afectivos y conativos (Schewe y Noble, 2000; Bruwer, Saliba y Miller, 2011). Por último, aunque la elección de la muestra fue intencional, en las dos universidades de las Islas Canarias se eligieron al azar días y horarios entre los de mayor asistencia a las aulas, de modo que, tras solicitar las autorizaciones correspondientes y explicar las instrucciones a los estudiantes, el cuestionario se aplicó a los sujetos que en ese momento estaban en el aula.

Se utilizó el cuestionario diseñado ad hoc como instrumento de recogida de información, tal y como es habitual en la literatura sobre las variables incluidas en este trabajo (Paiva, Sandoval y Bernardin, 2012). Para llevar a cabo el diseño de la escala se procedió primero al análisis de la literatura para identificar las variables, relaciones y medidas más apropiadas para el modelo propuesto, garantizando así la validez de contenido (Roy, Dewit y Aubert, 2001). Se tuvo en cuenta que en la literatura reciente se miden las variables incluidas en este estudio mediante un reducido número de ítems, evitando con ello los problemas metodológicos y los costes derivados del uso de múltiples indicadores (Bergkvist y Rossiter, 2007). Después de un pretest y siguiendo los principios de brevedad y simplicidad se obtuvo la escala definitiva tipo Likert de 14 ítems, con 5 alternativas de respuesta (1: en absoluto o nada de acuerdo, 5: totalmente de acuerdo). Los constructos analizados con la escala son: apego al lugar (4 ítems), familiaridad (2 ítems), identidad de lugar (4 ítems), satisfacción ( 2 ítems) y lealtad ( 2 ítems). Además, en el cuestionario se solicitaba al sujeto el sexo, la isla de residencia y el grado en el que ha realizado turismo en la región.

Desde el punto de vista metodológico en la investigación se han utilizado las ecuaciones estructurales, que constituyen un avance respecto a las técnicas de regresión (Falk y Miller, 1992). Existen dos modelos o métodos de ecuaciones estructurales (MEE): por una parte están los MEE basados en la covarianza (MBC) y en el análisis factorial, cuyos cálculos se llevan a cabo con programas informáticos como LISREL, AMOS y EQS; y por otra parte se encuentran los MEE basados en el estudio de la varianza, los mínimos cuadrados ordinarios (OLS) y el análisis de componentes principales (ACP), que se estiman con PLS (Partial Least Squares). El análisis PLS y el MBC deben ser considerados métodos complementarios más que competitivos (Chin et al., 2003).

Tal y como se evidencia en la literatura acerca de la lealtad en turismo en este trabajo se ha optado por el uso de PLS (Baptista, Campón y Hernández, 2013). Las razones son que el método PLS: (i) posee un potencial predictivo óptimo (Chin y Newsted, 1999; Cepeda y Roldán, 2004; Barroso, Cepeda y Roldán, 2005, 2010); (ii) aporta soluciones igualmente fiables que el análisis de covarianzas pero con menores restricciones (Falk y Miller, 1992; Chin et al., 2003); (iii) los procedimientos matemáticos y estadísticos subyacentes en el sistema son rigurosos y robustos (Wold, 1979); (iv) permite el uso de un amplio rango de tamaños muestrales (Medina, Rufín y Rey, 2011); (v) maximiza la varianza explicada (Chin y Newsted, 1999; Barroso, Cepeda y Roldán, 2010; Hair, Ringle, y Sarstedt, 2011); (vi) refleja las condiciones teóricas y empíricas de las ciencias sociales, en las que las teorías no están suficientemente asentadas y es escasa la información disponible (Wold, 1979; Cepeda y Roldán, 2004); (vii) y porque el análisis PLS permite el uso de indicadores reflectivos, como es este el caso a tenor de las aportaciones de Jarvis, Mackenzie y Podsakoff (2003). 


\section{ANÁLISIS DE RESULTADOS}

El modelo PLS se divide a su vez en un modelo de medida, que relaciona las variables observables y su variable latente, y en un modelo estructural, que relaciona unas variables latentes con otras (Gutiérrez, Bulchand, Díaz y Parra, 2013).

El análisis del modelo de medida conlleva estudiar si los conceptos teóricos están medidos correctamente a través de las variables observadas (ítems). Ello implica el estudio de la fiabilidad y de la validez de las relaciones entre las variables observadas y las variables latentes a las que están asociadas. El estudio de la fiabilidad individual del ítem se lleva a cabo observando la carga factorial $(\lambda)$, es decir, las correlaciones simples de los indicadores con el constructo que pretenden medir. Realizado este proceso se puso de manifiesto que las variables observadas alcanzaron el nivel mínimo requerido $(\lambda \geq$ 0.70) (cuadro 3), motivo por el cual se aceptó que los indicadores formaban parte de sus correspondientes constructos.

\section{Cuadro 3}

\section{MODELO DE MEDIDA: DATOS BÁSICOS}

\begin{tabular}{|c|c|c|c|c|}
\hline Constructo & Ítem & Carga $\lambda$ & FC (alfa) & AVE \\
\hline \multirow{4}{*}{$\begin{array}{l}\text { Identidad de } \\
\text { lugar }\end{array}$} & ID1 Me siento identificado con Canarias & 0,843932 & \multirow{4}{*}{$\begin{array}{c}0,906318 \\
(0,862494)\end{array}$} & \multirow{4}{*}{0,707975} \\
\hline & ID2 Soy canario por mis vivencias y mi pasado & 0,796440 & & \\
\hline & ID3 Considero que formo parte de Canarias & 0,905252 & & \\
\hline & ID4 Mi vida está hecha en Canarias & 0,816014 & & \\
\hline \multirow{4}{*}{$\begin{array}{l}\text { Apego al } \\
\text { lugar }\end{array}$} & AP1 Me gusta vivir en Canarias & 0,832290 & \multirow{4}{*}{$\begin{array}{c}0,866752 \\
(0,799491)\end{array}$} & \multirow{4}{*}{0,619733} \\
\hline & AP2 Me fastidiaría tener que irme de Canarias & 0,781100 & & \\
\hline & AP3 Siento apego por Canarias & 0,731280 & & \\
\hline & AP4 Estoy emocionalmente unido a Canarias & 0,800836 & & \\
\hline \multirow[t]{2}{*}{ Familiaridad } & FA1 Canarias me es familiar & 0,783164 & \multirow{2}{*}{$\begin{array}{c}0,817889 \\
(0,562207)\end{array}$} & \multirow{2}{*}{0,692590} \\
\hline & FA2 Conozco Canarias como destino turístico & 0,878541 & & \\
\hline \multirow[t]{2}{*}{ Satisfacción } & SA1 Hacer turismo Canarias me genera satisfacción & 0,893098 & \multirow{2}{*}{$\begin{array}{c}0,894250 \\
(0,763689) \\
\end{array}$} & \multirow{2}{*}{0,808734} \\
\hline & SA2 Hacer turismo en Canarias cumple mis expectativas & 0,905453 & & \\
\hline \multirow[t]{2}{*}{ Lealtad } & LE1 Recomendaría Canarias a otras personas & 0,851692 & \multirow{2}{*}{$\begin{array}{c}0,864229 \\
(0,687554)\end{array}$} & \multirow{2}{*}{0,761018} \\
\hline & LE2 Volvería a hacer turismo en Canarias & 0,892556 & & \\
\hline
\end{tabular}

Fuente: elaboración propia.

Por otra parte, el estudio de la fiabilidad compuesta (FC), un indicador similar al alfa de Conbrach más adecuado que éste en el marco de las ecuaciones estructurales, demostró que, al estar todos los valores por encima de 0,70, el modelo de medida era internamente consistente y que todos los indicadores o variables observadas estaban midiendo su correspondiente variable latente (Hair, Ringle, y Sarsted, 2011).

También se analizó la validez convergente y la validez discriminante. Para evaluar la validez convergente del modelo se calculó la varianza extraída media (AVE), que proporciona información sobre la cantidad de varianza que un constructo obtiene de sus indicadores con relación a la cantidad de varianza debida al error de medida. En todos 
los casos el resultado fue superior a 0,50 , por lo que se constató que más del $50 \%$ de la varianza del constructo era debida a sus indicadores (Chin, 2010) (cuadro 3).

Respecto a la validez discriminante, ésta supone que cada constructo es significativamente diferente del resto de los constructos, con los que no se encuentra relacionado según la teoría. Para calcular la validez discriminante y siguiendo a Fornell y Larcker (1981) se comprobó en primer lugar que la raíz cuadrada de varianza extraída (AVE) (en la diagonal del cuadro 4) era mayor que la varianza compartida entre el constructo y los otros constructos del modelo (los datos que no se encuentran en la diagonal del cuadro) (Chin, 2010).

\section{Cuadro 4}

\section{RAÍZ DEL AVE Y CORRELACIONES ENTRE CONSTRUCTOS}

\begin{tabular}{|l|c|c|c|c|c|}
\hline Constructos & Identidad & Apego & Familiaridad & Satisfacción & Lealtad \\
\hline Identidad & $\mathbf{0 , 8 4 1 4 1 3}$ & & & & \\
\hline Apego & 0,634373 & $\mathbf{0 , 7 8 7 2 3 1}$ & & & \\
\hline Familiaridad & 0,375831 & 0,265062 & $\mathbf{0 , 8 3 2 2 2 0}$ & & \\
\hline Satisfacción & 0,353777 & 0,450250 & 0,437182 & $\mathbf{0 , 8 9 9 2 9 6}$ & \\
\hline Lealtad & 0,319528 & 0,318287 & 0,527083 & 0,639946 & $\mathbf{0 , 8 7 2 3 6 3}$ \\
\hline
\end{tabular}

Fuente: elaboración propia.

Adicionalmente, para analizar la validez discriminante se obtuvo también la matriz de cargas factoriales cruzadas (Chin, 1998b) (cuadro 5). Las cargas factoriales o correlaciones de Pearson de los indicadores con su propio constructo debían ser mayores que las mantenidas con el resto de constructos, como así se constató. Por tanto, los indicadores estaban más correlacionados con su propio constructo que con los otros.

Los resultados pusieron de manifiesto que el modelo de medida tenía una aceptable fiabilidad y validez convergente y discriminante.

\section{Cuadro 5}

\section{CARGAS FACTORIALES CRUZADAS}

\begin{tabular}{|l|c|c|c|c|c|c|}
\hline Constructo & Ítems & Identidad & Apego & Familiaridad & Satisfacción & Lealtad \\
\hline \multirow{4}{*}{ Identidad de lugar } & ID1 & $\mathbf{0 , 8 4 3 9 3 2}$ & 0,490950 & 0,385611 & 0,332388 & 0,293450 \\
\cline { 2 - 7 } & ID2 & $\mathbf{0 , 7 9 6 4 4 0}$ & 0,472874 & 0,203218 & 0,174842 & 0,170103 \\
\cline { 2 - 7 } & ID3 & $\mathbf{0 , 9 0 5 2 5 2}$ & 0,597566 & 0,369496 & 0,341215 & 0,307211 \\
\cline { 2 - 7 } & ID4 & $\mathbf{0 , 8 1 6 0 1 4}$ & 0,561043 & 0,278936 & 0,313276 & 0,281478 \\
\hline \multirow{4}{*}{ Apego al lugar } & AP1 & 0,465067 & $\mathbf{0 , 8 3 2 2 9 0}$ & 0,119683 & 0,279988 & 0,189341 \\
\cline { 2 - 7 } & AP2 & 0,444845 & $\mathbf{0 , 7 8 1 1 0 0}$ & 0,118971 & 0,322414 & 0,121098 \\
\cline { 2 - 7 } & AP3 & 0,524401 & $\mathbf{0 , 7 3 1 2 8 0}$ & 0,141454 & 0,253706 & 0,100826 \\
\cline { 2 - 7 } & AP4 & 0,542722 & $\mathbf{0 , 8 0 0 8 3 6}$ & 0,372009 & 0,493484 & 0,476292 \\
\hline Familiaridad & FA1 & 0,387377 & 0,227830 & $\mathbf{0 , 7 8 3 1 6 4}$ & 0,319942 & 0,286229 \\
\cline { 2 - 7 } & FA2 & 0,258711 & 0,217318 & $\mathbf{0 , 8 7 8 5 4 1}$ & 0,401323 & 0,560287 \\
\hline
\end{tabular}




\begin{tabular}{|l|c|c|c|c|c|c|}
\hline Satisfacción & SA1 & 0,190047 & 0,398355 & 0,350914 & $\mathbf{0 , 8 9 3 0 9 8}$ & 0,570717 \\
\cline { 2 - 7 } & SA2 & 0,439125 & 0,411230 & 0,433148 & $\mathbf{0 , 9 0 5 4 5 3}$ & 0,580212 \\
\hline \multirow{2}{*}{ Lealtad } & LE1 & 0,298040 & 0,316531 & 0,435624 & 0,505538 & $\mathbf{0 , 8 5 1 6 9 2}$ \\
\cline { 2 - 7 } & LE2 & 0,262909 & 0,244980 & 0,481885 & 0,605175 & $\mathbf{0 , 8 9 2 5 5 6}$ \\
\hline
\end{tabular}

Fuente: elaboración propia.

Respecto a la evaluación del modelo estructural se comprobó que, salvo en el caso del apego, las variables predictoras contribuían a explicar la varianza de la variable lealtad de manera significativa, pues los coeficientes path $(\beta)$ (pesos de regresión estandarizados) alcanzaron niveles adecuados y superiores al nivel óptimo $(\beta \geq 0.3)$ (Chin, 1998a, 1998b) (cuadro 6). Además, en todas las relaciones causales directas, excepto las que vinculan la identidad con la satisfacción y el apego con la lealtad, el estadístico $t$ obtuvo niveles que constataron la alta significatividad de las mismas $(\mathrm{P}<0,05)$, tal y como se puso de manifiesto en el análisis bootstrapping con 500 sub-muestras y 200 casos llevado a cabo (Gutiérrez, Bulchand, Díaz y Parra, 2013). La relación entre la identidad y la satisfacción, así como la del apego con la lealtad, no alcanzan los niveles mínimos aceptables $(\beta \geq 0,30)$.

\section{Cuadro 6 \\ EFECTOS, SIGNIFICATIVIDAD Y CONFIRMACIÓN DE HIPÓTESIS}

\begin{tabular}{|l|r|r|c|}
\hline Variables latentes & \multicolumn{1}{|c|}{ Path $(\boldsymbol{\beta})$} & \multicolumn{1}{|c|}{ T } & CH \\
\hline H1 Identidad $\rightarrow$ Familiaridad & 0,375831 & $4,728219^{*}$ & SI \\
\hline H2 Identidad $\rightarrow$ Apego & 0,634373 & $11,715431 * *$ & SI \\
\hline H3 Identidad $\rightarrow$ Satisfacción & $\mathbf{- 0 , 0 0 5 1 6 6}$ & $\mathbf{0 , 0 6 8 1 8 3}$ ns & NO \\
\hline H4 Familiaridad $\rightarrow$ Satisfacción & 0,343010 & $5,106292 *$ & SI \\
\hline H5 Apego $\rightarrow$ Satisfacción & 0,362608 & $5,342385 *$ & SI \\
\hline H6 Satisfacción $\rightarrow$ Lealtad & 0,501425 & $7,037389 * *$ & SI \\
\hline H7 Familiaridad $\rightarrow$ Lealtad & 0,304756 & $4,661168 *$ & SI \\
\hline H8 Apego $\rightarrow$ Lealtad & $\mathbf{0 , 0 1 1 7 4 1}$ & $\mathbf{0 , 1 6 8 4 7 9}$ ns & NO \\
\hline
\end{tabular}

$*$ nivel $\mathrm{p}<0,05 * *$ nivel $\mathrm{p}<0,01$

Fuente: elaboración propia.

Por tanto, existe una directa y significativa relación causal entre la identidad de lugar y la familiaridad $(\beta=0,376)$, y especialmente entre la identidad y el apego $(\beta=0,643)$. La relación de la familiaridad y del apego con la satisfacción también es significativa ( $\beta$ $=0,343$ y $\beta=0,363$ respectivamente), aunque no entre la identidad y la satisfacción ( $\beta$ $=-0.005)$. La satisfacción y la familiaridad influyen directamente en la formación de la lealtad del joven residente $(\beta=0,501$ y $\beta=0,305$ respectivamente), no sucediendo lo mismo en el caso del apego $(\beta=0,012)$. Estos resultados nos permiten confirmar seis de las primeras ocho hipótesis, a excepción de las hipótesis tres (H3) y la hipótesis ocho (H8).

En el estudio del modelo estructural se calcularon tres indicadores adicionales (cuadro 7): (i) el indicador $\mathrm{R}^{2}$, que informa acerca de la cantidad de varianza explicada en cada 
variable latente dependiente; (ii) el indicador $\mathrm{Q}^{2}$, desarrollado por Stone (1974) y Geisser (1975) para medir la relevancia predictiva de los constructos dependientes; y (iii) el test GoF (Goodness-of-Fit), que representa la media geométrica entre la media del indicador AVE y la media de $\mathrm{R}^{2}$ en relación a los constructos endógenos (Wetzels, OdekerkenSchröder y Van Oppen, 2009). Se constató que las variables latentes precedentes explicaron suficiente varianza de las variables consecuentes, pues el indicador básico $\mathrm{R}^{2}$ alcanzó en todos los casos, excepto en el de la familiaridad, valores por encima del nivel mínimo aceptable $\left(\mathrm{R}^{2} \geq 0.30\right)$ (Falk y Miller, 1992), siendo en el caso de la familiaridad su valor inferior al nivel 0,19 propuesto por Chin (1998a). Por su parte, los valores superiores a cero del indicador $\mathrm{Q}^{2}\left(\mathrm{Q}^{2} \geq 0\right)$ permitieron constatar la relevancia predictiva del modelo (Riquel y Vargas, 2013). Por último, se obtuvo un valor de GoF de 0,4914, que es superior al valor mínimo aceptable $(\mathrm{GoF} \geq 0.36)$ considerando la situación más desfavorable para este test, que es el de muestras con efectos elevados (Wetzels, Odekerken-schröder y Van Oppen, 2009) (cuadro 7).

\section{Cuadro 7}

INDICADORES R ${ }^{2}, Q^{2}$ Y GoF

\begin{tabular}{|l|c|c|c|}
\hline Constructo & $\mathbf{R}^{\mathbf{2}}$ & $\mathbf{Q}^{\mathbf{2}}\left(^{*}\right)$ & AVE \\
\hline Apego al lugar & 0,402429 & 0,363151 & 0,619733 \\
\hline Familiaridad & 0,141249 & 0,133689 & 0,692590 \\
\hline Satisfacción & 0,311395 & 0,375408 & 0,808734 \\
\hline Lealtad & 0,485253 & 0,271498 & 0,761018 \\
\hline Media & ------ & $\mathbf{0 , 7 2 0 5 1 9}$ & $\mathbf{0 , 3 3 5 0 8 1}$ \\
\hline GoF & ------ & & $\mathbf{0 , 4 9 1 4}$ \\
\hline
\end{tabular}

(*) Este test es una medida de hasta qué punto los valores observados son reproducidos por el modelo y por sus parámetros estimados.

Fuente: elaboración propia

Los datos del cuadro 8 relativos al análisis descriptivo permiten afirmar que los niveles de todas las variables son altos. El 29\% de los ítems obtuvo una puntuación mínima de 2 y todos los ítems obtuvieron valoraciones iguales o superiores al $72 \%$ del valor máximo posible $(678 \times 5=3390)$ si todos los sujetos $(\mathrm{N}=678)$ hubieran valorado con la máxima puntuación (5) los diferentes reactivos. Por consiguiente se confirma la hipótesis novena (H9).

\section{Cuadro 8 \\ ESTADÍSTICOS DESCRIPTIVOS}

\begin{tabular}{|l|c|c|r|r|r|r|}
\hline Ítems & Mín & Máx & Suma & \% & Media & DT \\
\hline ID1 Me siento identificado con Canarias & 1 & 5 & 2472 & $72,92 \%$ & 3,65 & 1,174 \\
\hline ID2 Soy canario por mis vivencias y mi pasado & 1 & 5 & 2670 & $78,76 \%$ & 3,94 & 1,208 \\
\hline ID3 Considero que formo parte de Canarias & 1 & 5 & 2797 & $82,51 \%$ & 4,13 & 1,074 \\
\hline ID4 Mi vida está hecha en Canarias & 1 & 5 & 2811 & $82,92 \%$ & 4,15 & 1,091 \\
\hline AP1 Me gusta vivir en Canarias & 2 & 5 & 3072 & $90,62 \%$ & 4,53 & 0,722 \\
\hline
\end{tabular}




\begin{tabular}{|l|c|c|c|r|r|r|}
\hline AP2 Me fastidiaría tener que irme de Canarias & 1 & 5 & 2196 & $64,78 \%$ & 3,24 & 1,282 \\
\hline AP3 Siento apego por Canarias & 1 & 5 & 2557 & $75,43 \%$ & 3,77 & 1,006 \\
\hline AP4 Estoy emocionalmente unido a Canarias & 2 & 5 & 2931 & $86,46 \%$ & 4,32 & 0,798 \\
\hline FA1 Canarias me es familiar & 1 & 5 & 2889 & $85,22 \%$ & 4,26 & 0,905 \\
\hline FA2 Conozco Canarias como destino turístico & 1 & 5 & 2740 & $80,83 \%$ & 4,04 & 1,031 \\
\hline SA1 Hacer turismo Canarias me genera satisfacción & 1 & 5 & 2656 & $78,35 \%$ & 3,92 & 1,039 \\
\hline $\begin{array}{l}\text { SA2 Hacer turismo en Canarias cumple mis } \\
\text { expectativas }\end{array}$ & 1 & 5 & 2578 & $76,05 \%$ & 3,80 & 1,048 \\
\hline LE1 Recomendaría Canarias a otras personas & 3 & 5 & 3249 & $95,84 \%$ & 4,79 & 0,499 \\
\hline LE2 Volvería a hacer turismo en Canarias & 2 & 5 & 2952 & $87,08 \%$ & 4,35 & 0,482 \\
\hline Ítem adicional: He hecho turismo en Canarias & 3 & 5 & 3057 & $90,18 \%$ & 4,51 & 0,475 \\
\hline
\end{tabular}

Fuente: elaboración propia.

Por último, para contrastar la hipótesis 10 (H10) se llevaron a cabo dos análisis diferentes. En primer lugar se efectuó un análisis discriminante, un método que además de permitir pronosticar a qué grupo pertenecerá un determinado sujeto, también facilita la identificación de diferencias significativas en las respuestas de los grupos y en función de los criterios deseados (Redondo y Rodríguez, 2014). Los resultados del cuadro 9 permiten afirmar que no existen diferencias significativas en las respuestas directamente aportadas por los jóvenes residentes en Canarias, ni por razones de sexo, ni por de isla de residencia (Tenerife o Gran Canaria) ni por la cantidad de viajes realizados en Canarias. Esto queda demostrado por los reducidos niveles de los auto-valores y de la correlación canónica, así como los niveles próximos a uno del indicador Lambda, con una significatividad elevada $(\mathrm{P} \leq 0,005)$. Estos resultados permiten confirmar la primera parte de la décima hipótesis (H10) del trabajo.

\section{Cuadro 9}

\section{ANÁLISIS DISCRIMINANTE}

\begin{tabular}{|c|c|c|c|c|c|c|c|c|}
\hline \multicolumn{2}{|c|}{ Por provincia de residencia } & \multicolumn{3}{c|}{ Por sexo } & \multicolumn{3}{c|}{ Por cantidad de viajes } \\
\hline AV & CC & LW & AV & CC & LW & AV & CC & LW \\
\hline 0,140 & 0,114 & 0,966 & 0,011 & 0,102 & 0,990 & 0,029 & 0,168 & 0,972 \\
\hline \multicolumn{3}{|c|}{ Sig. $=0,000$} & \multicolumn{3}{c|}{ Sig. $=0,000$} \\
\hline \multicolumn{3}{|c|}{ AV: autovalor CC: correlación canónica LW: lambda de Wilks } \\
\hline
\end{tabular}

Fuente: elaboración propia.

Ahora bien, es posible que no existan diferencias significativas en las respuestas directas que los subgrupos dan al cuestionario, como así ha sucedido, pero que si se produzcan diferencias entre grupos que estén relacionadas con el modelo causal. En este sentido y en el contexto de la metodología de las ecuaciones estructurales, más concretamente mediante el método PLS, es posible llevar a cabo un análisis multi-grupo de diversas maneras. La fórmula más laboriosa consiste en analizar de manera independiente y por separado para cada grupo, tanto el modelo de medida como el modelo estructural. Llevado a cabo este análisis utilizando el criterio de sexo se constató la 
fiabilidad y la validez del modelo de medida para ambos grupos, el de hombres y el de mujeres, con valores similares entre ellos y en relación al modelo de medida obtenido para el conjunto de la muestra. También se puso de manifiesto que los dos modelos estructurales resultantes poseían similares relaciones directas (paths) entre ellos y en relación al modelo estructural obtenido para el conjunto muestral, tanto en valores absolutos como en significatividad. Lo mismo sucedió con los indicadores $\mathrm{Q}^{2}, \mathrm{R}^{2}$ y GoF. No obstante, se debe tener en cuenta que el análisis por separado de los grupos no permite determinar la significatividad de las posibles diferencias entre los modelos (el de medida y el estructural) de cada grupo (en nuestro caso hombres y mujeres), ni entre aquellos y los relativos al conjunto de la muestra.

Para poder realizar dicho análisis es necesario hacer uso de alguno de los métodos desarrollados por diversos autores, como es el caso del método paramétrico, entre otros (Chin, 2000; Henseler et al., 2007; Byrne, 2008). En este trabajo hemos estudiado las diferencias causales entre hombres y mujeres utilizando el método de análisis multigrupo denominado PLS-MGA, que ha sido desarrollado por Henseler et al. (2009) y Sarstedt et al. (2011) mediante procedimientos bootsrapping. A través de dicho método se calcularon las diferencias en las relaciones paths entre ambas sub-muestras, hombre y mujeres, y también se determina la significatividad de dichas relaciones. Los resultados utilizando 5.000 casos y un nivel de significatividad del 0,05 se recogen en el cuadro 10 .

\section{Cuadro 10 \\ ANÁLISIS MULTIGRUPO}

\begin{tabular}{|l|r|r|}
\hline Variables latentes & $\begin{array}{c}\text { Path }(\beta) \\
\text { diferencias }\end{array}$ & \multicolumn{1}{|c|}{ P-value } \\
\hline H1 Identidad $\rightarrow$ Familiaridad & 0,175 & $0,011^{*}$ \\
\hline H2 Identidad $\rightarrow$ Apego & 0,231 & $0,000^{*}$ \\
\hline H3 Identidad $\rightarrow$ Satisfacción & 0,008 & 0,097 \\
\hline H4 Familiaridad $\rightarrow$ Satisfacción & 0,177 & $0,976^{*}$ \\
\hline H5 Apego $\rightarrow$ Satisfacción & 0,229 & 0,949 \\
\hline H6 Satisfacción $\rightarrow$ Lealtad & 0,052 & 0,752 \\
\hline H7 Familiaridad $\rightarrow$ Lealtad & 0,292 & $0,004^{*}$ \\
\hline H8 Apego $\rightarrow$ Lealtad & 0,014 & 0,589 \\
\hline
\end{tabular}

Fuente: elaboración propia.

Teniendo en cuenta que se consideran significativas las diferencias de path con $\mathrm{P} \leq 0,05$ y $\mathrm{P} \geq 0,95$, se puede afirmar que no se confirma plenamente la segunda parte de la hipótesis 10 (H10), pues determinadas relaciones causales entre variables latentes (path) son significativamente diferentes entre el grupo de hombres y el grupo de mujeres. Específicamente son diferentes los efectos directos de la identidad sobre la familiaridad y el apego, así como de la familiaridad sobre la satisfacción y la lealtad. Sin embargo no alcanzan niveles de significatividad las diferencias respecto al efecto de la satisfacción sobre la lealtad y del apego sobre la lealtad. 


\section{CONCLUSIONES}

En conclusión, comprender y conseguir la lealtad del turista es una necesidad estratégica para las empresas del sector turístico, debido a la rivalidad existente en los mercados, a las crecientes demandas de los turistas y a los beneficios que la lealtad aporta, todo ello en un marco de desarrollo turístico sostenible y responsable. Las empresas deben asumir que la lealtad del turista debe ser un objetivo estratégico ligado a un futuro deseable, por referirse a la intención declarada de visitar nuevamente el destino o de recomendarlo. Por consiguiente, la sostenibilidad económica del destino turístico y de las empresas del sector está estrechamente vinculada a la consecución de la lealtad. Más específicamente es imprescindible estudiar y conseguir la lealtad del joven residente en los destinos formados por islas, tal y como se ha hecho en este trabajo, porque en la literatura se constata las peculiaridades de estos destinos tan poco analizados, así como el crecimiento y la importancia de este segmento tan escasamente estudiado.

En el caso de los jóvenes residentes de 18 y 19 años, los resultados de este estudio permiten conocer mejor el proceso por el que se genera la lealtad en este segmento que se comporta como los adultos y consume con cierta autonomía y frecuencia productos y servicios turísticos, deportivos y de ocio. Esto es relevante teniendo en cuenta que en el desarrollo turístico sostenible del destino los jóvenes residentes aportan información, conviven con los turistas foráneos, trabajan en las empresas que acogen a dichos turistas, ejercen el relevo generacional y aportan ingresos que ayudan a disminuir el carácter estacional de la demanda.

Se ha constatado en la literatura que la orientación al mercado, la reputación y la confianza de la empresa turística, así como la calidad, el valor y la imagen del productodestino, constituyen variables de la empresa y del producto que determinan la formación de la lealtad en el sector turístico. Ahora bien, también podemos concluir que las variables que relacionan al joven residente con el propio destino son suficientes para explicar la formación de su satisfacción y de su lealtad. En este sentido, las tres variables que hemos estudiado y que más han arraigado en la literatura, como son el apego al lugar, la familiaridad y la identidad de lugar (Rollero y De Piccoli, 2010), explican junto a la satisfacción la formación de la lealtad, sin intervención de las variables de la empresa y del producto. Esta conclusión es relevante por al menos dos razones. En primer lugar, el apego, la familiaridad y la identidad no constituyen variables específicamente vinculadas a la empresa o al producto, siendo el papel de la empresa en la formación de dichas variables poco determinante, pues la formación de dichas variables está asociada a la duración de la estancia y a las experiencias vividas durante dicha estancia. Esto significa que las empresas y las instituciones turísticas asociadas al destino turístico pueden conseguir la satisfacción y la lealtad del joven residente sin desarrollar acciones relacionadas con la orientación al mercado, con la empresa y con el producto-destino, pues de ello se encargan la identidad, el apego y la familiaridad.

No obstante y en segundo lugar, las actuaciones que las entidades turísticas del destino pudieran llevar a cabo para mejorar la orientación, la reputación y la confianza de la empresa, así como la calidad, el valor y la imagen del producto-destino, consiguiendo con ello más lealtad, tal y como se ha constatado en la literatura, debieran basarse en el 
fomento del apego, la familiaridad y la identidad del joven residente. Con ello las empresas conseguirían sinergias y una más sólida satisfacción y lealtad de los jóvenes que residen en el destino, además de asumir más responsabilidad social y fomentar la sostenibilidad. En este sentido las instituciones turísticas públicas y privadas se encuentran con un sustrato adecuado para el desarrollo de dichas actuaciones y para fomentar la lealtad de joven residente, pues se ha demostrado en este trabajo que el segmento ya posee un alto apego, familiaridad, identidad, satisfacción y lealtad, aunque dichos niveles pudieran ser aún más elevados.

Aunque en la revisión de la literatura no parezca clara la diferencia ni la relación entre la identidad, el apego y la familiaridad (Lewicka, 2008), en este trabajo se ha demostrado que se trata de constructos diferentes, que están relacionados y que de ellos derivan comportamientos de compra, tal y como han propuesto algunos autores (Jorgensen y Stedman, 2001) y como se deriva del contenido de los ítems utilizados. Se comprueba que la identidad se caracteriza por incorporar elementos especialmente cognitivos, como el auto-concepto, mientras que el apego al lugar incluye, sobre todo, componentes afectivos. La familiaridad se relaciona con el conocimiento, el aprendizaje y la experiencia del joven residente respecto al propio destino (Lewicka, 2010). Conocer estas diferencias entre los constructos y las implicaciones que las mismas tienen en el comportamiento del joven residente permite tender un puente entre los destinos turísticos, que son concebidos como lugares significativos, y la lealtad, sin la intervención directa de las variables de la empresa y del producto.

Se ha conseguido operativizar el apego, la familiaridad y la identidad de lugar, por más que en la literatura se constate la dificultad de medir dichos constructos a través de escalas, debido a que los ítems incluyen elementos tan variados como el self, el apego emocional, el significado del lugar, la pertenencia, los lazos sociales, los lazos generacionales y el significado espiritual (Low y Altman, 1992). Esto permite disponer de un marco de trabajo para desarrollar otros estudios referidos al mismo segmento de población en otros destinos turísticos, o en el mismo destino turístico utilizando otros segmentos de diferente edad, pudiendo así llevar a cabo las comparaciones que fueran pertinentes.

Se concluye que la identidad de lugar es la variable que inicia la cadena de efectos directos e indirectos que culminan en la satisfacción y la lealtad del joven residente. Aunque una mayor identidad de lugar no repercute directamente en la satisfacción y en la lealtad, dicha influencia se consigue a través de la familiaridad y del apego, que en gran medida dependen de la identidad (Hwang, Lee y Chen, 2005). Respecto a la familiaridad, en el trabajo se constata que es un antecedente directo de la satisfacción y de la lealtad (Kim, Ferrín y Rao, 2008), habiéndose demostrado también que, en el caso de los jóvenes residentes, la satisfacción es la variable que más efecto directo ejerce sobre la lealtad, tal y como proponen otros autores para otros segmentos (Kozak, 2003). Este hecho está a su vez en sintonía con la idea de que el desarrollo de un vínculo positivo con el lugar es un requisito para el ajuste psicológico del sujeto, en términos de satisfacción (Williams y McIntyre, 2001).

Por otra parte, aunque algunos autores consideran que la edad juega un rol importante en la formación del apego, de la familiaridad y de la identidad, es sobre todo la duración de la estancia o de la residencia, así como las experiencias asociadas a dicha estancia, la que determina la formación de dichos constructos, teniendo en cuenta que el segmento estu- 
diado tenía 18 y 19 años de edad. Es decir, es más probable que un residente de 18 años posea mayores niveles de apego, familiaridad e identidad en relación al propio destino que un turista foráneo de 50 años que no haya residido en dicho destino. No obstante, también se ha demostrado en otros trabajos que una residencia o una estancia no muy prolongada en el destino pudiera fomentar el apego, la familiaridad y la identidad (Knez, 2005).

Desde un punto de vista descriptivo y considerando las respuestas directas que los sujetos han dado a los ítems del cuestionario relativos al apego, se puede afirmar que nuestros hallazgos están en sintonía con otros investigadores que consideran que no existen diferencias significativas por razones de sexo, no al menos en el caso de los jóvenes residentes. No obstante algunos autores destacan un mayor apego al lugar por parte de las mujeres, debido a su rol (Lewicka, 2005). Adicionalmente a lo anterior, la ausencia de diferencias por razones de isla de residencia y por cantidad de viajes realizados en el propio destino está en concordancia con numerosos estudios sociológicos que evidencian que las generaciones poseen patrones homogéneos cognitivos, afectivos y comportamentales que son uniformes y estables entre sus miembros, especialmente si conviven en una misma comunidad y lugar (Charters et al., 2011). Por tanto, es muy probable que los resultados hubieran sido similares si se hubiese elegido una muestra de jóvenes residentes en Canarias que cursaran otros estudios, o que se encontraran trabajando, o incluso teniendo algunos años más de edad, debido al carácter global y universal que posee el segmento generacional estudiado.

Ahora bien, aunque los modelos de medida y los modelos estructurales de los hombres y las mujeres analizados de manera independiente eran estadísticamente significativos y similares entre sí y a los obtenidos para el conjunto de la muestra, se han hallado diferencias significativas en algunas relaciones directas (paths) de los modelos estructurales de ambos grupos de sujetos, los hombres y las mujeres. Estos resultados sugieren que una futura línea de investigación. Ello permitiría estudiar con más detalle otras posibles diferencias por razones de sexo, también significativas, en determinados indicadores o relaciones que permitan precisar las actuaciones a llevar a cabo por las empresas turísticas en la gestión de la satisfacción y la lealtad de los jóvenes residentes. Estas líneas de investigación también podrían abordar otros criterios diferenciadores utilizados en este trabajo, como son la isla de residencia y la cantidad de viajes realizados en el propio destino.

En su conjunto el enfoque generacional y socio-cultural de este trabajo permite sugerir que es posible realizar, desde las empresas e instituciones turísticas vinculadas al destino, actuaciones homogéneas para todo el segmento de jóvenes residentes, concentrando esfuerzos y ahorrando recursos. A la vez permite plantear la posibilidad de generalizar los resultados y conclusiones obtenidas al mismo segmento residente en otros destinos de España, o de otros países, pues la globalización y el desarrollo de las comunicaciones han permitido dar un carácter también global y universal a las generaciones. En todo caso estas podrían constituir también futuras líneas de investigación.

El trabajo llevado a cabo no ha estado exento de alguna limitación. La principal limitación ha estado relacionada con la dificultad de obtener muestras adecuadas en un contexto geográfico formado por islas. Esta limitación se ha intentado solventar mediante la elección de dos sub-muestras de jóvenes residentes en las dos islas mayores de la región, las más habitadas, cada una de las cuales es representativa y es una capital de las dos provincias que forman el archipiélago. 


\section{AGRADECIMIENTOS}

Los autores desean agradecer a los decanos y profesores de las universidades de La Laguna y Las Palmas de Gran Canaria la colaboración prestada, sin la cual este trabajo no hubiera podido llevarse a cabo.

\section{BIBLIOGRAFÍA}

ALMEIDA, F., BALBUENA, A., CORTÉS, R. (2015): "Resident's attitudes towards the impacts of tourism", Tourism Management Perspectives, $\mathrm{n}^{\circ} 13$, pp. 33-40ANDRADES, L., SÁNCHEZ, M., PULIDO, J.I. (2013): “Differentiating competitiveness through tourism image assessment: An application to Andalucía (Spain)", Journal of Travel Research, $\mathrm{n}^{\circ} 52$ (1), pp. 68-81.BALOGLU, S. (2001): "Image variations of Turkey by familiarity index: Informational and experiential dimensions", Tourism Management, $\mathrm{n}^{\circ}$ 22, pp. 127-133.BAPTISTA, H.M., CAMPÓN, A.M., HERNÁNDEZ, J.M. (2011): "El estudio de la lealtad en el turismo: una revisión de la literatura", Book of proceedings vol. I - International Conference on Tourism \& Management Studies, Algarve, pp. 850-862.

BARROSO, C., CEPEDA, G., ROLDÁN, J. (2005): “Investigar en economía de la empresa: partial least squares o modelos basados en la covarianza?", I Workshop Luso-Espanhol sobre Metodologia para Investigação em Ciências Empresariais: Intodução à Técnica Partial Least Squares (PLS), Universidade da Beira Interior, pp. 1-17.

BARROSO, C., CEPEDA, G., ROLDÁN, J. L. (2010): Applying maximun likelihood and PLS on different sample sizes: Studies on SERVQUAL model and employee behavior model. En V. Esposito Vinzi et al. (eds.): “Hanbook of Partial Least Squares, Springer Hanbooks of Computational Statistics", Verlag Berlin Heidelberg, pp. 427-447,

BERGKVIST, L., ROSSITER, J. R. (2007): "The predictive validity of multiple-item vs. single item measures of the same construct", Journal of Marketing Research, $\mathrm{n}^{\circ}$ 44 (2), pp. 175-184. BIGNÉ, E., SÁNCHEZ, M. I., SÁNCHEZ, J. (2001): “Tourism image, evaluation variables and after purchase behaviour: inter-relationship", Tourism Management, $\mathrm{n}^{\circ} 22$ (6), pp. 607-616.

BRUWER J., SALIBA A., MILLER B. (2011): "Consumer behavior and sensory preference differences: implications for wine product marketing", Journal of Consumer Marketing, $\mathrm{n}^{\circ} 28(1)$, pp. 5-18.

BYRNE, B.M. (2008): “Testing for multigroup equivalence of a measuring instrument: A walk through the process", Psicothema, no 20(4), pp. 872-882

CARMELI, A., TISHLER, A (2005): "Perceived organizational reputation and organizational performance: An empirical investigation of industrial enterprises", Corporate Reputation Review, n ${ }^{\circ}$ 8, pp. 13-30.

CEPEDA, G., ROLDÁN, J.L. (2004): “Aplicando en la práctica la técnica PLS en la administración de empresas”. In Conocimiento y Competitividad. XIV Congreso Nacional ACEDE. Murcia (pp. 74-8).

CHARTERS S. et al. (2011): "Generation Y and sparkling wines: A cross-cultural perspective", International Journal of Wine Business Research, $\mathrm{n}^{\mathrm{o}} 23$ (2), pp. 161-175. 
CHI, C. G., QU, H. (2008): "Examining the structural relationships of destination image, tourist satisfaction and destination loyalty: An integrated approach", Tourism Management, $\mathrm{n}^{\circ} 29$ (4), pp. 624-636.

CHIN, W.W. (1998a): "The Partial Least Approach to structural equation modelling". En G.A. Marcoulides (Ed.): "Moderm methods for business research". Mahwah: Lawrence Erlbaum Associates, pp.295-336.

CHIN, W.W. (1998b): "Issues and opinion on structural equation modeling", MIS Quarterly, no 22 (1), pp. 7-16.

CHIN, W.W. (2000): “Frequently Asked Questions - Partial Least Squares \& PLSGraph”. Home Page (Online). Available: http://disc-nt.cba.uh.edu/chin/plsfaq.htm

CHIN, W.W. (2010): "How to write up and report PLS analyses". En V. E. Vinzi, W. W. Chin, J. Henseler, \& H. Wang (Eds.): "Handbook of partial least squares" (pp. 655690). London, New York: Springer.

CHIN, W.W., MARCOLIN, B.L., NEWSTED, P.R. (2003): “A partial least squares latent variable modeling approach for measuring interaction effects: results from a Monte Carlo simulation study and an electronic mail emotion/ adoption study", Information Systems Research, $\mathrm{n}^{\circ}$ 14(2), pp. 189-217.

CHIN, W.W., NEWSTED, P.R. (1999): "Structural equation modeling analysis with small samples using partial least squares”. En R. Hoyle (Ed.): "Statistical strategies for small samples research” (pp. 307-341). Sage Publications.

DRAPER, J., WOOSNAM, K.M., NORMAN, W.C. (2011): “Tourism use history: Exploring a new framework for understanding residents' attitudes toward tourism", Journal of Travel Research, $\mathrm{n}^{\circ} 50$ (1), pp. 64-77.

EURICO, S., VALLE, P., SILVA, J. (2015): “A model of graduates' satisfaction and loyalty in tourism Higher Education: The role of employability", Journal of Hospitality, Leisure, Sport \& Tourism Education, n ${ }^{\circ} 16$, pp. 30-42.

EXCELTUR-GOBIERNO DE CANARIAS (2013): “Estudio de Impacto Económico del Turismo: Impactur Canarias 2012”. Disponible en: http://www.exceltur.org/excel01/ contenido/portal/files/informe-impactur canarias2012.pdf.

FALK, R.F., MILLER, N.B. (1992): “A primer for soft modelling”. Akron: The University of Akron Press.

FORGAS, S., PALAU, R., SÁNCHEZ, J., CALLARISA, L.J. (2012): “Urban destination loyalty drivers and cross-national moderator effects: The case of Barcelona", Tourism Management, $\mathrm{n}^{\mathrm{o}}$ 33, pp. 1309-1320.

FORNELL, C., LARCKER, D. (1981): "Evaluating structural equation models with unobservable variables and measurement error", Journal of Marketing Research, $\mathrm{n}^{\mathrm{o}}$ 18(1), pp. 39-50.

GEISSER, S. (1975): “A predictive sample reuse method with applications", Journal of the American Statistical Association, $\mathrm{n}^{\circ} 70$, pp. 320-328.

GROSS, M. ., BROWN, G. (2008): “An empirical structural model of tourists and places: progressing involvement and place attachment into tourism", Tourism Management, n 29 (6), pp. 1141-1151.

GUPTA, S., KIM, H. (2010): "Value-driven internet shopping: The mental accounting theory perspective", Psychology and Marketing, nº 27 (1), pp. 13-35. 
GUTIÉRREZ, D. (2010): "Las actitudes de los residentes ante el turismo". Tesis doctoral. La Laguna: Universidad de La Laguna.

GUTIÉRREZ, D., BULCHAND, J., DÍAZ, R., PARRA, E. (2013): “Antecedentes del uso de los medios sociales por el turista: motivación, oportunidad y capacidad", Cuadernos de Turismo, $\mathrm{n}^{\circ}$ 31, pp. 153-173.

HAIR, J.F., RINGLE, C.M., SARSTEDT, M. (2011): "PLS-SEM: Indeed a silver bullet", Journal of Marketing Theory and Practice, $\mathrm{n}^{\circ} 19$ (2), pp. 139-151.

HENSELER, J., MARTENS, H., NAES, T. (2007): “A new and simple approach to multigroup analysis in partial least squares path modeling”. Proceedings of PLS'07: 5th International Symposium on PLS and Related Methods, 7, 104-107.

HENSELER, J., RINGLE, C.M., SINKOVICS, R. R. (2009): "The use of partial least squares path modeling in international marketing", Advances in International Marketing, $\mathrm{n}^{\circ} 20$, pp. 277-320.

HERNÁNDEZ, B., HIDALGO, M. C., SALAZAR-LAPLACE, M.E., HESS, S. (2007): "Place attachment and place identity in natives and non-natives", Journal of Environmental Psychology, no 27, pp. 310-319.

HIDALGO, M.C., HERNÁNDEZ, B. (2001): "Place attachment: Conceptual and empirical questions", Journal of Environmental Psychology, no 21 (3), pp. 273-281.

HWANG, S.N., LEE, C., CHEN, H.J. (2005): “The Relationship among tourists' involvement, place attachment and interpretation satisfaction in Taiwan's national parks", Tourism Management, $\mathrm{n}^{\mathrm{o}} 26$, pp. 143-156.

INSCH, A., FLOREK, M. (2008): “A great place to live, work and play: Conceptualizing place satisfaction in the case of a city's residents", Journal of Place Management and Development, $\mathrm{n}^{\circ} 1$ (2), pp. 138-149.

JAAFAR, M., NOOR, S., RASOOLIMANESH, S.M. (2015): "Perception of young local residents toward sustainable conservation programs: A case study of the Lenggong World Cultural Heritage Sit”, Tourism Management, $\mathrm{n}^{\circ}$ 48, pp. 154-163.

JARVIS, CH.B., MACKENZIE, S.B., PODSAKOFF, P.M. (2003): "Critical review of construct indicators and measurement model misspecification in marketing research", Journal of Consumer Research, $\mathrm{n}^{\circ}$ 30, pp. 199-218.

JORGENSEN, B.S., STEDMAN, R.C. (2001): "Sense of place as an attitude: lakeshore owners' attitudes toward their properties", Journal of Environmental Psychology, $\mathrm{n}^{\circ}$ 21, pp. 233-248.

KASSIM, N., ABDULLAH, N.A. (2010): “The effect of perceived service quality dimensions on customer satisfaction, trust, and loyalty in e-commerce settings: A cross cultural analysis", Asia Pacific Journal of Marketing and Logistics, $\mathrm{n}^{\circ} 22$ (3), pp. 351-371.

KIM, D. ., FERRÍN, D.L., RAO, H.R. (2008): “A trust-based consumer decision-making model in electronic commerce: the role of trust, perceived risk, and their antecedents", Decision Support Systems, $\mathrm{n}^{\circ} 44$ (2), pp. 544-564.

KNEZ, I. (2005): “Attachment and identity as related to a place and its perceived climate", Journal of Environment Psychology, n⿳ 25, pp. 207-218

KOHLI, A.K., JAWORSKI, B.J. (1990): "Market orientation: the construct, research propositions and managerial implications", Journal of Marketing Research, $\mathrm{n}^{\circ} 54$ (2), pp. 1-18. 
KOZAK, M. (2003): "Measuring tourist satisfaction with multiple destinations attributes", Tourism Analysis, $\mathrm{n}^{\circ}$ 7, pp. 229-240.

KYLE, G., GRAEFE, A., MANNING, R., BACON, J. (2004a): "Effect of activity involvement and place attachment on recreationists' perceptions of setting density", Journal of Leisure Research, $\mathrm{n}^{\circ} 36$ (2), pp. 209-231.

KYLE, G., GRAEFE, A., MANNING, R., BACON, J. (2004b): "Predictors of behavioral loyalty among hikers along the Appalachian Trail", Leisure Sciences, no 26 (1), pp. 99-118.

LASZLO, C., SHERMAN, D., ELLISON, J. (2005): "Expanding the value horizon: how stakeholder value contributes to competitive advantage", Journal of Corporate Citizenship, $\mathrm{n}^{\mathrm{o}} 20$, pp. 65-76.

LEE, J., GRAEFE, A.R., BURNS, R. C. (2007): "Examining the antecedents of destination loyalty in a forest setting", Leisure Sciences, n 29 (5), pp. 463-481.

LEE, T.H. (2013): "Influence analysis of community resident support for sustainable tourism development", Tourism Management, $\mathrm{n}^{\circ}$ 34, pp. 37-46.

LEWICKA, M. (2005): "Ways to make people active: Role of place attachment, cultural capital and neighborhood ties", Journal of Environmental Psychology, n 4, pp. 381-395.

LEWICKA, M. (2008): "Place attachment, place identity, and place memory: restoring the forgotten city past", Journal of Environmental Psychology, n ${ }^{\circ} 28$, pp. 209-231.

LEWICKA, M. (2010): "What makes neighborhood different from home and city? Effects of place scale on place attachment", Journal of Environmental Psychology, n 30, pp. $35-51$.

LOW, S.M., ALTMAN, I. (1992): "Place attachment: A conceptual inquiry". In I. Altman y S. M. Low (Eds.): "Place attachment” (pp. 1-12). New York and London: Plenum Press.

LUO, M., FENG, R., CAI, L.A. (2008): "Information search behavior and tourist characteristics", Journal of Travel \& Tourism Marketing, $\mathrm{n}^{\circ} 17$ (2), pp. 15-25.

MARTÍNEZ, J.A. (2014): “Comportamiento de compra del turista residente. El caso de la generación " $Y$ ” en Canarias". Tesis doctoral. La Laguna: Universidad de La Laguna.

MCKERCHER, B., DENIZCI, B. (2010): “Are tourists or markets destination loyal”, Journal of Travel Research, $\mathrm{n}^{\circ} 50$ (2), pp. 121-132.

MCKERCHER, B., DENIZCI, B., NG,E. (2012): "Rethinking loyalty", Annals of Tourism Research, $\mathrm{n}^{\circ} 39$ (2), pp. 708-734.

MEDINA, C., RUFÍN, R., REY, M. (2011): "El papel moderados de la cultura en la generación de satisfacción y lealtad", Investigaciones Europeas de Dirección y Economía de la Empresa, $\mathrm{n}^{\circ}$ 17(1), pp. 57-73.

MEYDEU, A., LADO, N. (2003): "Market orientation and business economic Performance", International Journal of Service Industry Management, $\mathrm{n}^{\circ} 14$ (3), pp. 284-309.

NARVER, J. C., SLATER, S.F. (1990): “The effect of market orientation on business profitability", Journal of Marketing Research, no 54 (4), pp. 20-35.

NUSAIR, K. K., PARSA, H.G., COBANOGLU, C. (2011): "Building a model of commitment for Generation Y: An empirical study on e-travel retailers", Tourism Management, $\mathrm{n}^{\circ} 32$ (4), pp. 833-843. 
OREJA, J. R., PARRA, E., YANES, V. (2008): “The sustainability of island destinations: tourism area life cycle and teleological perspectives. The case of Tenerife", Tourism Management, $\mathrm{n}^{\circ} 29$, pp. 53-65.

PAIVA, G., SANDOVAL, M., BERNARDIN, M. (2012): "Factores explicativos de la lealtad de clientes de los supermercados", Innovar, n 22(44), pp. 153-164.

PEARCE, P.L., KANG, M. H. (2009): "The effects of prior and recent experience on continuing interest in tourism settings", Annals of Tourism Research, n ${ }^{\circ} 36(2)$, pp. 172-190.

REDONDO, D., RODRÍGUEZ, J.M. (2014): "Crisis en las entidades de crédito españolas: un estudio mediante análisis discriminante", Estudios de Economía Aplicada, ${ }^{\circ}$ 32(2), pp. 617-644.

RIQUEL, F.J., VARGAS, A. (2013): “Las presiones institucionales del entorno medioambiental: aplicación a los campos de golf”, Revista Europea de Dirección y Economía de la Empresa, $\mathrm{n}^{\circ} 22$, pp. 29-38.

ROLLERO, C., DE PICCOLI, N.D. (2010): "Place attachment, identification, and environment perception: An empirical study", Journal of Environmental Psychology, ${ }^{\circ}$ 30, pp. 198-205.

ROY, M.C., DEWIT, O., AUBERT, B.A. (2001): “The impact of interface usability on trust in Web retailers", Internet Research, no 11(5), pp. 388-398.

SÁNCHEZ, J.L., GARCÍA, A., MARCHANTE, A.J. (2014): “Análisis de la incidencia de las certificaciones de calidad sobre la productividad de los hoteles en Andalucía”, Universia Business Review, no 44, pp. 88-103.

SARSTEDT, M., HENSELER, J., RINGLE, C.M. (2011): "Multi-group analysis in partial least squares (pls) path modeling: Alternative methods and empirical results", Advances in International Marketing, $\mathrm{n}^{\mathrm{o}} 22$, pp. 195-218.

SCHEWE, C.D., NOBLE, S.M. (2000): "Marketing segmentation by cohorts: The value and validity of cohorts in America and abroad", Journal of Marketing Management, $\mathrm{n}^{\mathrm{o}} 16(1)$, pp. 129-142.

SCHEYVENS, R., MOMSEN, J. (2008): “Tourism and poverty reduction: Issues for small island states", Tourism Geographies, n 10(1), 22-41.

SCOTT, D., VITARTAS, P. (2008): “The Role of involvement and attachment in satisfaction with local government services", International Journal of Public Sector Management, $\mathrm{n}^{\mathrm{o}} 21(1), \mathrm{pp} .45-57$.

SHARPLEY, R. (2014): "Host perceptions of tourism: A review of the research", Tourism Management, $\mathrm{n}^{\circ} 42$, pp. 37-49.

STONE, M. (1974): "Cross-validator choice and the assessment of statistical predictions (with discussion)", Journal of the Royal Statistical society, Ser. B, no 36, pp. 111-133.

SUN, X., CHI, CH., XU, H. (2013): "Developing destination loyalty: the case of Hainan Island", Annals of Tourism Research, $\mathrm{n}^{\circ}$ 43, pp. 547-577.

UM, S., CHON, K., RO, Y. (2006): “Antecedents of revisit intention”, Annals of Tourism Research, $\mathrm{n}^{\circ} 33$ (4), pp. 1141-1158.

WANG, S., XU, H. (2015): "Influence of place-based senses of distinctiveness, continuity, self-esteem and self-efficacy on residents' attitudes toward tourism", Tourism Management, $\mathrm{n}^{\circ} 47$, pp. 241-250 
WETZELS, M., ODEKERKEN-SCHROEDER, G., VAN OPPEN, C. (2009): “Using PLS path modelling for assessing hierarchical construct models: Guidelines and empirical illustration", MIS Quarterly, no 33(1), pp. 177-195.

WILLIAMS, D.R., MCINTYRE, N. (2001): "Where heart and home reside: Changing constructions of place and identity". In: Trends 2000: Shaping the Future (September 17-20, 2000) (pp. 392-403).

WOLD, H. (1979): "Model construction and evaluation when theoretical knowledge is scarce: An example of the use of partial least squares". Cahiers du Département D’Économétrie. Genève: Faculté des Sciences Économiques et Sociales, Université de Genève.

WONG, I.A., WAN, Y.K.P. (2013): “A systematic approach to scale development in tourist shopping satisfaction: linking destination attributes and shopping experience", Journal of Travel Research, $\mathrm{n}^{\circ}$ 52(1), pp. 29-41.

WOODSIDE, A.G., LYSONSKI, S. (1989): “A general model of traveler destination choice", Journal of Traveler Research, $\mathrm{n}^{\circ}$ 27(4), pp. 8-14.

WU, M. Y., PEARCE, P.L. (2013): "Tourists to Lhasa, Tibet: how local youth classify, understand and respond to different types of travelers", Asia Pacific Journal of Tourism Research, $\mathrm{n}^{\circ}$ 18(6), pp. 549-572.

YUKSEL, A., YUKSEL, F., BILIM, Y. (2009): "Destination attachment: effects on customer satisfaction and cognitive, affective and conative loyalty", Tourism Management, $\mathrm{n}^{\circ} 31(2)$, pp. 274-284.

ZHANG, H., FU, X., CAI, L.A., LU, L. (2014): "Destination image and tourist loyalty: A meta-analysis", Tourism Management, n 40, pp. 213-223. 